\title{
Observation of quantum-measurement backaction with an ultracold atomic gas
}

\author{
Kater W. Murch ${ }^{1}$, Kevin L. Moore ${ }^{1}$, Subhadeep Gupta ${ }^{1}$, and Dan M. Stamper-Kurn ${ }^{1,2}$ \\ ${ }^{1}$ Department of Physics, University of California, Berkeley CA 94720 \\ ${ }^{2}$ Materials Sciences Division, Lawrence Berkeley National Laboratory, Berkeley, CA 94720 \\ (Dated:)
}


Current research on micro-mechanical resonators strives for quantum-limited detection of the motion of macroscopic objects [1]. Prerequisite to this goal is the observation of measurement backaction consistent with quantum metrology limits [2]. However, thermal noise presently dominates measurements and precludes ground-state preparation of the resonator. Here we establish the collective motion of an ultracold atomic gas confined tightly within a Fabry-Perot optical cavity [3, 4, 5] as a system for investigating the quantum mechanics of macroscopic bodies. The cavity-mode structure selects a single collective vibrational mode that is measured by the cavity's optical properties, actuated by the cavity optical field, and subject to backaction by the quantum force fluctuations of this field. Experimentally, we quantify such fluctuations by measuring the cavity-light-induced heating of the intracavity atomic ensemble. These measurements represent the first observation of backaction on a macroscopic mechanical resonator at the standard quantum limit.

Various types of micro-mechanical resonators, including singly [6, 7] or doubly [8, 9, 10] clamped nanofabricated beams, thin membranes [11], and toroidal structures [12], have been fabricated and used to study small-amplitude vibrations. With resonance frequencies in the $\mathrm{kHz}$ to $\mathrm{MHz}$ range - an exception being the $\mathrm{GHz}$ resonator of Ref. [13] - these resonators remain significantly perturbed by thermal noise at cryogenic temperatures. Nevertheless, powerful schemes to cool a single mechanical mode of the resonator below its ambient temperature have been demonstrated [6, 7, 8, $9,10,11,12]$. These schemes use either active feedback or the passive dynamical backaction on a driven resonator, the latter being equivalent to cavity-induced laser cooling of atoms [14, 15]. The use of these schemes to achieve ground-state cooling has been discussed [16, 17, 18].

In this work, we demonstrate that the collective motion of a trapped macroscopic ensemble of ultracold atoms may serve as the resonator for the study of quantum micro-mechanics. In contrast with the mechanical systems discussed above, such atoms may be cooled directly to the ground state of motion. Non-classical states of motion have been engineered in atomic ensembles [19], and the oscillatory motion of an atomic gas has been used to measure weak forces [20], analogous to measurements using microfabricated cantilevers [21]. However, previous efforts have lacked the means to measure the motion of an atomic ensemble at the quantum limit. 
High-finesse optical cavities have been used to sense the motion of single atoms [22, 23]. Their sensitivity results from the spatial variation of the atom-cavity coupling frequency; in a near-planar Fabry-Perot cavity, this frequency varies as $g(z)=g_{0} \sin k_{p} z$ along the cavity axis, where $k_{p}$ is the wavevector of light near the cavity resonance. In the case where the detuning $\Delta_{c a}=\omega_{c}-\omega_{a}$ between the bare-cavity (no atoms present) and the atomic resonance frequencies is large $\left(\left|\Delta_{c a}\right| \gg\left\{g_{0}, \Gamma\right\}\right)$, a single atom of half-linewidth $\Gamma$ at position $z$ causes the cavity resonance to be shifted by $g^{2}(z) / \Delta_{c a}$. Measuring the cavity resonance thus provides information on the atom's position.

Such a measurement may be applied also to monitor the motion of an ensemble of $N$ atoms that are optically trapped within the resonator mode. In this case, a single collective degree of freedom couples exclusively to a single mode of the cavity (see supplemental information). For small displacements of the atoms from their potential minima, we define a collective position operator $Z=\left(N_{\text {eff }}\right)^{-1} \sum_{i} \sin \left(2 k_{p} \bar{z}_{i}\right) \delta z_{i}$, and the conjugate momentum $P=\sum_{i} \sin \left(2 k_{p} \bar{z}_{i}\right) p_{i}$, with $\bar{z}_{i}$ being the equilibrium position of the $i^{\text {th }}$ atom, $\delta z_{i}$ its position deviation from equilibrium operator, and $p_{i}$ being its momentum. The cavity then serves to monitor a specific collective mode of motion in the atomic ensemble, with the cavity resonance being shifted by $\Delta_{N}-N_{\text {eff }} f_{0} Z / \hbar$ where $\Delta_{N}=\sum_{i} g^{2}\left(\bar{z}_{i}\right) / \Delta_{c a}$ is the cavity frequency shift with all atoms localized at their potential minima and $f_{i}=-\hbar \partial_{z} g^{2}\left(\bar{z}_{i}\right) / \Delta_{c a}=$ $f_{0} \sin \left(2 k_{p} \bar{z}_{i}\right)$ is the optical dipole force from a single cavity photon. That is, the collective mode sensed by the cavity is equivalent to the center-of-mass motion of $N_{\text {eff }}=\sum_{i} \sin ^{2}\left(2 k_{p} \bar{z}_{i}\right)$ atoms trapped at locations of maximum sensitivity of the cavity properties to the atomic position.

With the identification of the collective variables $Z$ and $P$, we may draw directly on results obtained for the motion of radiation-pressure-driven mechanical resonators within optical cavities. For example, we conclude that optical dipole forces in a driven cavity will displace the collective variable $Z$, shifting the cavity resonance frequency and leading to cavity optical nonlinearity and bistability [3, 24]. We find also that force fluctuations arising from the quantum fluctuations of the intracavity optical field disturb the collective momentum $P$ and constitute the quantum backaction for cavity-based measurements of the displacement $Z[25]$.

To assess the impact of these dipole force fluctuations, we consider the dynamics of the atoms-cavity system with the cavity continuously driven by laser light of fixed detuning 
$\Delta_{p c}$ from the bare-cavity resonance. The average optical force of $\bar{n}$ cavity photons displaces the collective position variable by $\Delta Z=\left(\hbar k g_{0}^{2} / m \omega_{z}^{2} \Delta_{c a}\right) \bar{n}$ and thereby shifts the cavity resonance frequency to $\omega_{c}^{\prime}=\omega_{c}+\Delta_{N}-N_{\text {eff }} f_{0} \Delta Z / \hbar$, where $\omega_{z}$ is the trap frequency. We define collective quantum operators $a$ and $a^{\dagger}$ through the relations $Z-\Delta Z=Z_{\mathrm{ho}}\left(a^{\dagger}+a\right)$ and $P=i P_{\mathrm{ho}}\left(a^{\dagger}-a\right)$, with $Z_{\mathrm{ho}}=\sqrt{\hbar / 2 m \omega_{z} N_{\mathrm{eff}}}$ and $P_{\mathrm{ho}}=\hbar /\left(2 Z_{\mathrm{ho}}\right)$. As discussed in the supplemental information, we obtain equations of motion for $a$ and for the cavity field operator $b$ as

$$
\begin{aligned}
& \frac{d a}{d t}=-i \omega_{z} a+i \kappa \epsilon(n-\bar{n}), \\
& \frac{d b}{d t}=-i \omega_{c}^{\prime} b+i \kappa \epsilon\left(a^{\dagger}+a\right) b-\kappa b+\sqrt{2 \kappa} b_{i n}
\end{aligned}
$$

where $\kappa$ is the decay rate of the cavity field, $b$ is the cavity photon annihilation operator and $b_{i n}$ represents the coherent-state input field that drives the cavity.

Here, we introduce a dimensionless "granularity" parameter, $\epsilon=N_{\text {eff }} f_{0} Z_{\mathrm{ho}} /(\hbar \kappa)$, that quantifies the coupling between quantum fluctuations of the collective atomic and optical fields. In the non-granular regime, defined by $\epsilon \ll 1$, the generally complex atoms-cavity dynamics described by Eqs. 1 and 2 are vastly simplified. To characterize this regime, consider the impulse $N_{\text {eff }} f_{0} / 2 \kappa$ imparted upon the collective motion by the single photon optical force over the $(2 \kappa)^{-1}$ lifetime of a cavity photon. For $\epsilon \ll 1$, this impulse is smaller than the zero-point momentum fluctuations of rms magnitude $P_{\text {ho }}$; thus, the effects of optical force fluctuations on the atomic ensemble are adequately described by coarse graining. Likewise, the transient displacements induced by this impulse will shift the cavity resonance by an amount that is much smaller than $\kappa$; thus, the quantum fluctuations of the cavity-optical field are the same as in the absence of the intracavity atomic gas, with the spectral density of photon number fluctuations being $S_{n n}(\omega)=2 \bar{n} \kappa\left(\kappa^{2}+(\Delta+\omega)^{2}\right)^{-1}$ [16] with $\Delta=\Delta_{p c}-\left(\omega_{c}^{\prime}-\omega_{c}\right)$ being the probe detuning from the atoms-shifted cavity resonance.

We then find the occupation number of the collective atomic excitation to vary as

$$
\frac{d}{d t}\left\langle a^{\dagger} a\right\rangle=\kappa^{2} \epsilon^{2}\left[S_{n n}^{(-)}+\left(S_{n n}^{(-)}-S_{n n}^{(+)}\right)\left\langle a^{\dagger} a\right\rangle\right]
$$

where $S_{n n}^{( \pm)}=S_{n n}\left( \pm \omega_{z}\right)$ and we assume $\left\langle a^{\dagger} a\right\rangle$ remains small. The collective atomic motion is subject to momentum diffusion, which heats the atomic gas at a per-atom rate of $R_{\mathrm{c}}=$ $\hbar \omega_{z} \kappa^{2} \epsilon^{2} S_{n n}^{(-)} / N$, and also to coherent damping or amplification of the atomic motion [14, 15, 16, 17]. 


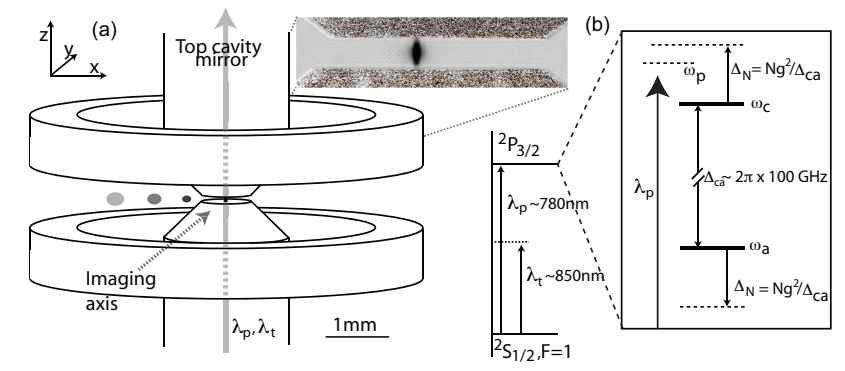

FIG. 1: (a) Ultracold atoms are produced in a magnetic trap, formed using electromagnets coaxial with the vertically oriented high-finesse cavity, and delivered to the cavity center. Trapping/locking light $\left(\lambda_{t}=850 \mathrm{~nm}\right)$ and probe light $\left(\lambda_{p}=780 \mathrm{~nm}\right)$ are sent through the cavity and monitored in transmission. An absorption image, obtained using probe light along the $\hat{y}$ axis, shows atoms trapped optically within the cavity volume. (b) Energy level scheme for the far-detuned $\left(\Delta_{c a} \gg\right.$ $\left.\sqrt{N} g_{0}\right)$ cavity.

So far we have neglected the force fluctuations on the atoms associated with incoherent scattering. As in free space, spontaneous emission by atoms driven by laser light leads to momentum diffusion due to both recoil kicks and fluctuations of the optical dipole force [26, 27]. Allowing the trapped atoms to be distributed evenly along the cavity axis, the total light-induced per-atom heating rate becomes $R=R_{\mathrm{fs}}+R_{\mathrm{c}}$ where $R_{\mathrm{fs}}=\left(f_{0}^{2} / 2 m\right)(\bar{n} / \kappa)(1 / C)$ is the free-space diffusive heating rate in a standing wave of light [26, 27]. For this atomic distribution, $N_{\text {eff }}=N / 2$ and we obtain $R_{\mathrm{c}}=R_{\mathrm{fs}} \times C\left(1+\left(\Delta-\omega_{z}\right)^{2} / \kappa^{2}\right)^{-1}$. Thus, in the strong coupling regime of cavity quantum electrodynamics, with single-atom cooperativity $C=g_{0}^{2} / 2 \kappa \Gamma \gg 1$, diffusive heating may be dominated by backaction heating $\left(R_{\mathrm{c}}\right)$ for probe frequencies near the cavity resonance $\left(\left|\Delta-\omega_{z}\right|<\kappa\right)$.

In our experiment, this backaction heating was measured bolometrically. Because the mechanical $Q$ of the collective vibrational mode is low ( $\sim 40$ as determined in Ref. [3] ), backaction induced excitation of this mode soon leads to a rise in the total thermal energy of the atomic sample. We quantify this energy increase by measuring the evaporative loss of trapped atoms from a finite-depth optical trap. By using an ultracold atomic gas, with temperature $T \ll \hbar \kappa / k_{B}$, the coherent amplification or damping of collective motion [see Eq. 3. may be neglected, and the atom heating rate is related directly to the spectral density of photon fluctuations in the cavity.

For this heating measurement, we prepared an ultracold gas of ${ }^{87} \mathrm{Rb}$ atoms within a 
high-finesse Fabry-Perot optical resonator [3] (Fig. 1). One TEM 00 mode of the cavity was excited resonantly with light at wavevector $k_{t}=2 \pi /(850 \mathrm{~nm})$. This light, far detuned from atomic resonances, formed a one-dimensional optical lattice of depth $U / k_{B}=6.6(7) \mu \mathrm{K}$ in which the atoms were trapped and evaporatively cooled to a temperature of $T=0.8 \mu \mathrm{K}$, as determined by time-of-flight measurements after the atoms were released from the trap. The atoms occupied approximately 300 adjacent sites in the optical lattice. Given $k_{B} T<\hbar \omega_{z}$, where $\omega_{z}=2 \pi \times 42 \mathrm{kHz}$ is the axial trap frequency in each lattice site, all axial vibration modes, including the collective mode pertinent to cavity-based position measurements, were cooled to their ground state. The atomic sample was probed using light with wavevector $k_{p}=2 \pi /(780 \mathrm{~nm})$ that was nearly resonant with another $\mathrm{TEM}_{00}$ mode of the cavity. For this light, the cavity mirrors, separated by $194 \mu \mathrm{m}$ and each with $5 \mathrm{~cm}$ radius of curvature, displayed measured losses and transmissions per reflection of 3.8 and $1.5 \mathrm{ppm}$, respectively, yielding $\kappa=2 \pi \times 0.66 \mathrm{MHz}$. The bare-cavity resonance frequency for this mode $\omega_{c}$ was maintained at a detuning of $\left|\Delta_{c a}\right|=2 \pi \times(30-100) \mathrm{GHz}$ from the ${ }^{87} \mathrm{Rb} \mathrm{D} 2$ atomic resonance. The cavity was stabilized by passive in vaccuo vibration isolation and by active feedback based on transmission measurements of the trapping light at wavevector $k_{t}$.

The atom-cavity coupling frequency $g_{0}=2 \pi \times 14.4 \mathrm{MHz}$ was determined from measured cavity parameters and by summing over all excitations from the $\left|F=1, m_{F}=-1\right\rangle$ hyperfine ground state by $\sigma^{+}$probe light on the D2 resonance line. With the atomic resonance halflinewidth being $\Gamma=2 \pi \times 3 \mathrm{MHz}$, the single-atom cooperativity of $C=g_{0}^{2} / 2 \kappa \Gamma=52$ satisfies the criterion for strong coupling.

To measure the backaction heating near the cavity resonance, $N=10^{5}$ atoms were loaded into the cavity, causing the cavity resonance to be shifted by $\Delta_{N}=2 \pi \times 100 \mathrm{MHz}$ at the atom-cavity detuning of $\Delta_{c a}=2 \pi \times 100 \mathrm{GHz}$. The cavity was then driven with probe light detuned by $\Delta_{p c}=2 \pi \times 40 \mathrm{MHz}$ from the bare-cavity resonance. Transmission through the cavity was monitored using single-photon counting devices. The cavity photon number $\bar{n}$ was obtained from the transmission signal using the measured quantum efficiency of $0.040(8)$ for detecting intracavity photons.

While the transmitted probe intensity was initially negligible owing to the large detuning between the probe and cavity resonance frequencies, the ongoing loss of atoms from the optical trap eventually brought the atoms-cavity resonance near the probe frequency, leading to discernible transmission (Fig. 2(a)). We used this transmission signal to determine the 


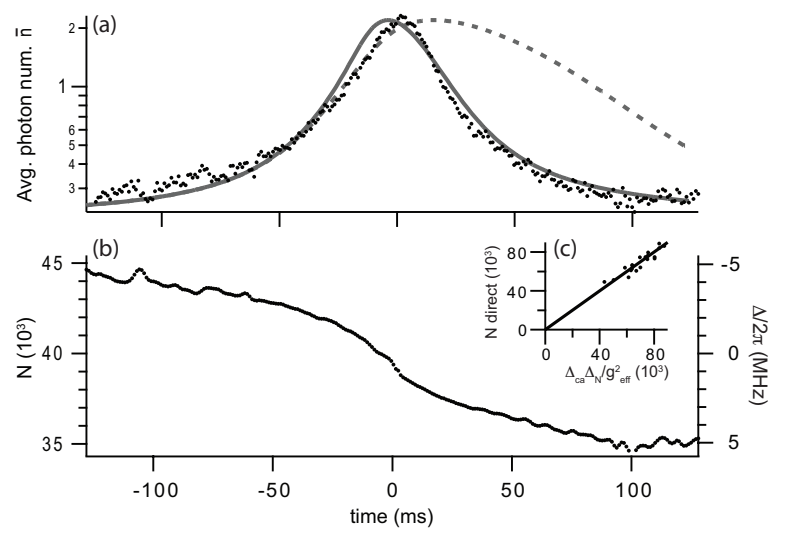

FIG. 2: Cavity-based observation of evaporative atomic losses due to cavity-light-induced diffusive heating. (a) The intracavity photon number, $\bar{n}$ (points, average of 30 measurements) is monitored as the atom number is reduced by evaporation, and the cavity resonance is brought across the fixed probe frequency. The expected $\bar{n}(t)$ excluding (dashed) or including (solid) cavity-enhanced diffusive heating are shown. (b) The atom number $N(t)$ is inferred from the measured photon number based on the cavity lineshape. Atoms are lost at a background rate of $0.9(1) \mathrm{s}^{-1}$ per atom away from the cavity resonance, and thrice faster near resonance. (c) The relation between $2 \Delta_{c a} \Delta_{N} /\left.g_{0}^{2}\right|_{Z=0}$ and the atom number measured directly by absorption imaging matches with predictions (line).

atom number $N$ and its rate of change $d N / d t$ as functions of time. We related $\Delta_{N}$ to the instantaneous transmitted probe power by assuming a Voigt lineshape for the cavity transmission with a Gaussian kernel of rms frequency width $\sigma=2 \pi \times 1.1 \mathrm{MHz}$ chosen to account for broadening due to technical fluctuations in $\Delta_{p c}$. We also modified the lineshape to account for the probe-induced displacements of the collective position $Z$ that were as high as $3.5 \mathrm{~nm}$ for the maximum cavity photon number $(\bar{n}=1.9)$ used here [3]. As shown in Fig. 2(b), the atom loss rate was strongly enhanced near resonance due to increased light-induced heating.

From the observed loss rate we determined the per-atom heating rate of the trapped atomic sample as $R=-U d(\ln N) / d t$ (Fig. 2, 3). Atoms experiencing intracavity intensity fluctuations of cavity-resonant light were heated at a per atom rate that is $R / R_{\mathrm{fs}} \simeq 40$ times larger than that of atoms exposed to a standing wave of light of equal intensity in free space. The cavity-induced heating was abated for light detuned from the cavity resonance. 


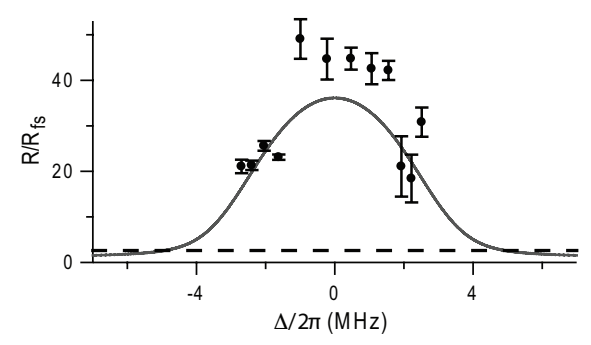

FIG. 3: Cavity-heating of a collective atomic mode in a strongly coupled Fabry-Perot cavity over spontaneous emission dominated free space heating. The measured ratio $R / R_{\mathrm{fs}}$ is shown with $1 \sigma$ statistical error bars. For each measurement, a 12 ms-long range of the probe transmission measurement data was used to determine $d N / d t$ and $\bar{n}$. Systematic errors, at a level of $23 \%$ at the cavity resonance, arise from uncertainty in the background loss rate, the background light level, and overall photo-detection efficiency. Grey line shows theoretical prediction (with no adjustable parameters) as described in the text. Dashed line shows an upper bound on the off-resonance heating rate based on measurements at $\Delta_{c a}=2 \pi \times 29.6 \mathrm{GHz}$ and $\Delta=2 \pi \times 40 \mathrm{MHz}$.

While this cavity-enhanced diffusion has been inferred from the lifetime [28] and spectrum [29] of single atoms in optical cavities, our measurements are performed under experimental conditions that allow its direct quantification.

To compare the observed heating rate with that expected based on quantum-measurement backaction (Fig. 3), we account for technical fluctuations in the probe detuning $\delta$ by a convolution of the predicted frequency dependence with the aforementioned Gaussian kernel. For $\delta=0$, this convolution reduces the measured heating rate per photon by a factor of 0.7 below what is expected in absence of technical fluctuations. The measured atom heating rates agree well with their predicted value, confirming that the backaction heating of the atomic ensemble is at the level required for quantum-limited measurements. Using the relation between $R_{\mathrm{c}}$ and $S_{n n}$, the measured heating rate may be interpreted as a measurement of the spectral density of intracavity photon number fluctuations where the atomic ensemble is used as a mechanical sensing medium for these fluctuations. From the measured maximum heating rate of $R / R_{\mathrm{fs}}=43(10)$, the error being predominantly systematic, and accounting for the convolved cavity line shape, we obtain the spectral noise power of photon fluctuations in a resonantly driven cavity as $S_{n n} / \bar{n}=4.0(9) \times 10^{-7} \mathrm{~s}$, in agreement with the predicted $S_{n n} / \bar{n}=2 / \kappa=4.8 \times 10^{-7} \mathrm{~s}$. 
We have shown that heating due to cavity-induced fluctuations of the optical dipole force dominates the heating of a trapped atomic gas near resonance. To highlight this finding further, we measured the atom heating rate due to intracavity light that is far from the cavity resonance, for which one should observe the spontaneous-emission-dominated heating of atoms in free space. For a atom-cavity detuning of $\Delta_{c a}=2 \pi \times 29.6 \mathrm{GHz}$ and $N \simeq 9000$ atoms, we excited the cavity for a variable time with probe light at detuning $\Delta=2 \pi \times 40$ $\mathrm{MHz}$ with an intracavity photon number of $\bar{n}=2$. From the decay rate of $N$, we observed a probe-light-induced per-atom loss rate that, if ascribed completely to diffusive heating of the atomic sample, yields a heating rate of $R / R_{\mathrm{fs}}=2.9(7)$, far smaller than that observed at the cavity resonance. Yet, these losses exceeded those expected based on diffusion from Rayleigh scattering. This discrepancy may be explained by additional effects of Raman scattering. Atoms scattered by the $\sigma^{+}$probe light into different hyperfine ground states couple to the cavity probe light with different strength, thereby changing the relationship between $\Delta_{N}$ and the atom number $N$. These additional effects appear sufficient to account for our observations, yet are constrained by our measurements to contribute only slightly to the atom losses observed from probe light at the cavity resonance.

This work demonstrates the bright prospects for studying quantum aspects of the motion of macroscopic $\left(N_{\text {eff }} \simeq 10^{5}\right.$ atoms $)$ mechanical systems. The optical confinement of ultracold atoms within a high-finesse optical resonator enabled the construction of a nearly ground state mechanical resonator. The identification, and quantitative measurement of cavity heating as the quantum backaction of position measurement on a collective mode of a mechanical object is a significant demonstration of the ultracold-atom approach to quantum micro-mechanics.

Working in the non-granular regime and with an atomic medium at sufficiently low temperatures so that cavity cooling/anti-cooling could be neglected, we may interpret the measured backaction heating as a direct measurement of the spectrum of photon fluctuations in a driven cavity, a quantity of fundamental interest in quantum optics. We note that these fluctuations are not visible in the coherent light transmitted through the cavity, for which the shot-noise spectrum remains white (see supplemental information). Specifically, in a cavity driven by coherent laser light, the atoms serve as an in-situ heterodyne detector of cavity-enhanced fluctuations of the electromagnetic field, with the two quadratures of collective motion serving as two heterodyne receivers at the beat frequency $\omega_{z}$. At present, 
by quantifying only the total heating rate of the trapped atomic gas, we cannot access information on the individual noise quadratures. However, augmented by time-resolved measurements of the collective motion, as demonstrated in Ref. [3], our setup may also serve to probe quadrature-squeezed light before the intracavity squeezing is degraded by attenuation outside the cavity [30].

We have demonstrated that atoms in a strong-coupling cavity are heated optically at a rate that exceeds that calculated for free-space illumination. This fact presents a challenge to cavity-aided non-destructive measurements of atom number or spin with uncertainty below the standard quantum limit [31, 32, 33, 34, 35, 36]. In such measurements, the sensitivity gained by increasing the probe light fluence is eventually offset by the increased disturbance of the atoms due to incoherent light scattering. Our work suggests that cavities with singleatom cooperativity beyond $C=1$ will yield benefits to these measurements only if the measurement is made insensitive to the atomic position, e.g. by placing atoms at antinodes of the cavity field or in traps for which $\omega_{z} \gg \kappa$.

We thank T. Purdy and S. Schmid for early contributions to the experimental apparatus, and S.M. Girvin, J. Harris, H.J. Kimble, H. Mabuchi, and M. Raymer for helpful discussions. This work was supported by AFOSR, DARPA, and the David and Lucile Packard Foundation. 


\section{Supplementary Information}

\section{Development of collective modes}

The atoms in our system are confined at many locations within a one dimensional optical lattice of wavevector $k_{t}=2 \pi / 850 \mathrm{~nm}$, yet interact with the cavity mode at the position dependent coupling rate $g(z)=g_{0} \sin k_{p} z$. Because of the position dependent coupling and the distribution of the atoms, a single collective degree of freedom (but not a center of mass degree of freedom) interacts with the cavity mode.

We consider that each atom is trapped harmonically with frequency $\omega_{z}$ and trap center $\bar{z}_{i}$, where we denote the displacement of atom $i$ from its trap's center by the operator $\delta z_{i}=$ $z_{i}-\bar{z}_{i}=z_{\mathrm{ho}}\left(\hat{a}_{i}^{\dagger}+\hat{a}_{i}\right)$ with $z_{\mathrm{ho}}=\sqrt{\hbar / 2 m \omega_{z}}$ being the harmonic oscillator length and atom field operators $\hat{a}_{i}$ and $\hat{a}_{i}^{\dagger}$ conventionally defined. We assume that the atomic displacements are small $\left(k_{p} \delta z_{i} \ll 1\right)$ and that the cavity-atom detuning is large $\left(\left|\Delta_{c a}\right| \gg g_{0} \sqrt{N}\right)$. Omitting some constant terms, we obtain a Hamiltonian describing the coupled atoms/cavity system as

$$
\mathcal{H}=\left(\hbar \omega_{c}+\sum_{i}\left[\frac{\hbar g^{2}\left(\bar{z}_{i}\right)}{\Delta_{c a}}-f_{i} \delta z_{i}\right]\right) n+\mathcal{H}_{a}+\mathcal{H}_{i n}
$$

where $n$ is the cavity photon number operator, $\mathcal{H}_{a}=\sum_{i} \hbar \omega_{z} \hat{a}_{i}^{\dagger} \hat{a}_{i}$, and $\mathcal{H}_{i n}$ describes optical modes external to the cavity and their coupling to the cavity field [37]. Here, the peratom cavity resonance shift is expanded to first order in the atomic position operator, with $f_{i}=-\hbar \partial_{z} g^{2}(z) / \Delta_{c a}=f_{0} \sin \left(2 k_{p} \bar{z}_{i}\right)$ being the optical dipole force on atom $i$ from a single cavity photon.

We define a collective position operator $Z=\left(N_{\text {eff }}\right)^{-1} \sum_{i} \sin \left(2 k_{p} \bar{z}_{i}\right) \delta z_{i}$, and the conjugate momentum $P=\sum_{i} \sin \left(2 k_{p} \bar{z}_{i}\right) p_{i}$, with $p_{i}$ being the momentum of atom $i$, and $N_{\text {eff }}=\sum_{i} \sin ^{2}\left(2 k_{p} \bar{z}_{i}\right)$. The cavity then serves to monitor a specific collective mode of motion in the atomic ensemble, with the cavity resonance being shifted by $\Delta_{N}-N_{\text {eff }} f_{0} Z / \hbar$ where $\Delta_{N}=\sum_{i} g\left(\bar{z}_{i}\right)^{2} / \Delta_{c a}$ is the cavity frequency shift with all atoms localized at their potential minima. 
Given these collective operators we can write equations of motion;

$$
\begin{aligned}
\dot{P} & =\sum_{i} \sin \left(2 k_{p} \bar{z}_{i}\right)\left(-m \omega_{z}^{2} \delta z_{i}+f_{i} n\right) \\
& =-N_{\text {eff }} m \omega_{z}^{2}\left(Z-\frac{f_{0} \bar{n}}{m \omega_{z}^{2}}\right)+f_{0}(n-\bar{n}) . \\
\dot{Z} & =\frac{P}{N_{\text {eff }} m} .
\end{aligned}
$$

A constant average optical force of $\bar{n}$ cavity photons displaces the collective position variable by $\Delta Z=\left(\hbar k g_{0}^{2} / m \omega_{z}^{2} \Delta_{c a}\right) \bar{n}$ and thereby shifts the cavity resonance frequency to $\omega_{c}^{\prime}=\omega_{c}+\Delta_{N}-N_{\text {eff }} f_{0} \Delta Z / \hbar$. We define collective quantum operators $a$ and $a^{\dagger}$ through the relations $Z-\Delta Z=Z_{\mathrm{ho}}\left(a^{\dagger}+a\right)$ and $P=i P_{\mathrm{ho}}\left(a^{\dagger}-a\right)$, with $Z_{\mathrm{ho}}=z_{\mathrm{ho}} / \sqrt{N_{\mathrm{eff}}}$ and $P_{\mathrm{ho}}=\hbar /\left(2 Z_{\mathrm{ho}}\right)$. With these substitutions, we have the Hamiltonian describing the collective mode-cavity system:

$$
\mathcal{H}=\hbar \omega_{c}^{\prime} n-N_{\mathrm{eff}} f_{0} Z_{\mathrm{ho}}\left(a^{\dagger}+a\right)(n-\bar{n})+\hbar \omega_{z} a^{\dagger} a+\mathcal{H}_{i n}
$$

\section{Calculation of the heating rate}

Given equation 8, we can draw directly on existing results which analyze cavity cooling and heating for similar Hamiltonians [16]. For clarity, however, we present a derivation of the heating rate below. From Eq. 8 we obtain equations of motion for $a$ and for the cavity field operator $b$.

$$
\begin{aligned}
& \frac{d a}{d t}=-i \omega_{z} a+i \kappa \epsilon(n-\bar{n}) \\
& \frac{d b}{d t}=-i \omega_{c}^{\prime} b+i \kappa \epsilon\left(a^{\dagger}+a\right) b-\kappa b+\sqrt{2 \kappa} b_{i n}
\end{aligned}
$$

where $\kappa$ is the decay rate of the cavity field and $b_{\text {in }}$ represents the coherent-state input field that drives the cavity. We have introduced the granularity parameter $\epsilon=N_{\text {eff }} f_{0} Z_{\mathrm{ho}} /(\hbar \kappa)$ as discussed in the text. We can now express the atomic field operator as,

$$
a(t)=e^{-i \omega_{z} t} a(0)+i \kappa \epsilon \int_{0}^{t} d t^{\prime} e^{-i \omega_{z}\left(t-t^{\prime}\right)}\left(n\left(t^{\prime}\right)-\bar{n}\right)
$$


From here, we evaluate the rate of change of the atomic energy:

$$
\begin{aligned}
\frac{d}{d t}\left(a^{\dagger} a\right) & =\left(\frac{d}{d t} a^{\dagger}\right)_{t} a(t)+a^{\dagger}(t)\left(\frac{d}{d t} a\right)_{t} \\
= & {\left[i \omega_{z} a^{\dagger}(t)-i \kappa \epsilon(n(t)-\bar{n})\right] a(t)+a^{\dagger}(t)\left[-\omega_{z} a^{\dagger}(t)+i \kappa \epsilon(n(t)-\bar{n})\right] } \\
= & 2 \kappa^{2} \epsilon^{2} \operatorname{Re}\left[\int_{0}^{t} d t^{\prime}(n(t)-\bar{n})\left(n\left(t^{\prime}\right)-\bar{n}\right) e^{-i \omega_{z}\left(t-t^{\prime}\right)}\right] \\
& \quad+i \kappa \epsilon\left(a^{\dagger}(0)(n(t)-\bar{n}) e^{i \omega_{z} t}-(n(t)-\bar{n}) a(0) e^{-i \omega_{z} t}\right) .
\end{aligned}
$$

For the sake of evaluating the cavity field evolution we restrict our treatment to times which are short compared to the timescale over which the atomic motion is significantly varied by interaction with the light. Under this ansatz we approximate Eq. 11 as

$$
a(t) \simeq e^{-i \omega_{z} t} a(0)
$$

Inserting this solution for the atomic field operator into the equation of motion for the cavity field, (2) we have the following for the frequency components of $b$ :

$$
-i \omega b(\omega)=-i \omega_{c}^{\prime} b(\omega)-\kappa b(\omega)+\sqrt{2 \kappa} b_{i n}(\omega)+i \kappa \epsilon\left(a(0) b\left(\omega-\omega_{z}\right)+a^{\dagger}(0) b\left(\omega+\omega_{z}\right)\right) .
$$

Defining $L(\omega)=\left(1-i\left(\omega-\omega_{c}^{\prime}\right) / \kappa\right)^{-1}$, we obtain

$$
b(\omega)=\frac{L(\omega)}{\kappa}\left[\sqrt{2 \kappa} b_{i n}(\omega)+i \epsilon\left(a(0) b\left(\omega-\omega_{z}\right)+a^{\dagger}(0) b\left(\omega+\omega_{z}\right)\right)\right] .
$$

We can solve this equation iteratively,

$$
\begin{aligned}
b(\omega)=\frac{L(\omega)}{\kappa}[ & \sqrt{2 \kappa} b_{i n}(\omega)+i \epsilon \sqrt{2 \kappa}\left(a(0) L\left(\omega-\omega_{z}\right) b_{i n}\left(\omega-\omega_{z}\right)\right. \\
& \left.\left.+a^{\dagger}(0) L\left(\omega+\omega_{z}\right) b_{i n}\left(\omega+\omega_{z}\right)\right)+\mathcal{O}\left(|\epsilon a(0)|^{2}\right)\right] .
\end{aligned}
$$

In the non-granular regime $\epsilon \ll 1$, and assuming small values of $a(0)$, i.e. that the atoms are sufficiently cold, we neglect terms of order $\epsilon^{3}$ or higher.

Returning to Eq. 14 we now have

$$
\begin{aligned}
n(t)= & \frac{1}{2 \pi} \int d \omega_{1} d \omega_{2} e^{i\left(\omega_{1}-\omega_{2}\right) t} b^{\dagger}\left(\omega_{1}\right) b\left(\omega_{2}\right) \\
= & \frac{1}{2 \pi} \int d \omega_{1} d \omega_{2} e^{i\left(\omega_{1}-\omega_{2}\right) t} \frac{L^{*}\left(\omega_{1}\right) L\left(\omega_{2}\right)}{\kappa^{2}} 2 \kappa\left[b_{i n}^{\dagger}\left(\omega_{1}\right) b_{i n}\left(\omega_{2}\right)+\right. \\
& i \epsilon b_{i n}^{\dagger}\left(\omega_{1}\right)\left(a(0) L\left(\omega_{2}-\omega_{z}\right) b_{i n}\left(\omega_{2}-\omega_{z}\right)+a^{\dagger}(0) L\left(\omega_{2}+\omega_{z}\right) b_{i n}\left(\omega_{2}+\omega_{z}\right)\right)- \\
& \left.i \epsilon\left(a^{\dagger}(0) L^{*}\left(\omega_{1}-\omega_{z}\right) b_{i n}^{\dagger}\left(\omega_{1}-\omega_{z}\right)+a(0) L^{*}\left(\omega_{1}+\omega_{z}\right) b_{i n}^{\dagger}\left(\omega_{1}+\omega_{z}\right)\right) b_{i n}\left(\omega_{2}\right)\right] .
\end{aligned}
$$


With the above normally ordered product of operators $b_{i n}$ we are justified in replacing:

$$
\begin{aligned}
& b_{i n}(\omega) \rightarrow \sqrt{\pi \kappa n_{\max }} \delta\left(\omega-\omega_{p}\right), \\
& b_{i n}^{\dagger}(\omega) \rightarrow \sqrt{\pi \kappa n_{\max }} \delta\left(\omega-\omega_{p}\right),
\end{aligned}
$$

where $\omega_{p}$ is the frequency of a probe laser, and $n_{\max }$ is the maximum intracavity photon number for resonant cavity excitation. Finally, we obtain

$$
\begin{array}{r}
n(t)=\bar{n}\left[1+i \epsilon\left(a(0) L\left(\omega_{p}+\omega_{z}\right) e^{-i \omega_{z} t}+a^{\dagger}(0) L\left(\omega_{p}-\omega_{z}\right) e^{+i \omega_{z} t}\right)-\right. \\
\left.i \epsilon\left(a^{\dagger}(0) L^{*}\left(\omega_{p}+\omega_{z}\right) e^{+i \omega_{z} t}+a(0) L^{*}\left(\omega_{p}-\omega_{z}\right) e^{-i \omega_{z} t}\right)\right] .
\end{array}
$$

Here we have substituted $\bar{n}=n_{\max }\left|L\left(\omega_{p}\right)\right|^{2}$.

We are now in a position to evaluate the heating rate:

$$
\begin{aligned}
& \frac{d}{d t} E= \hbar \omega_{z}\left\langle\frac{d}{d t} a^{\dagger} a\right\rangle \\
&=2 \hbar \omega_{z} \kappa^{2} \epsilon^{2} \operatorname{Re}\left[\int_{0}^{t} d t^{\prime}\left\langle(n(t)-\bar{n})\left(n\left(t^{\prime}\right)-\bar{n}\right)\right\rangle e^{-i \omega_{z}\left(t-t^{\prime}\right)}\right]+ \\
& i \hbar \omega_{z} \kappa \epsilon\left\langle a^{\dagger}(0)(n(t)-\bar{n}) e^{i \omega_{z} t}-(n(t)-\bar{n}) a(0) e^{-i \omega_{z} t}\right\rangle .
\end{aligned}
$$

Addressing the first term first; for a linear cavity driven by a constant coherent state input, we substitute the relation,

$$
\langle n(\tau) n(0)\rangle-\langle n(\tau)\rangle^{2}=\bar{n} e^{i\left(\omega_{p}-\omega_{c}^{\prime}\right) \tau-\kappa \tau} .
$$

Assuming the system is in a steady state, in that $\left\langle n(t) n\left(t^{\prime}\right)\right\rangle=\left\langle n\left(t-t^{\prime}\right) n(0)\right\rangle$, and substituting $\bar{n}^{2}=\langle n(\tau)\rangle^{2}$ we obtain for the first half of the heating rate,

$$
2 \hbar \omega_{z} \kappa \epsilon^{2} \bar{n}\left(\frac{1}{1+\left(\omega_{p}-\omega_{c}^{\prime}-\omega_{z}\right)^{2} / \kappa^{2}}\right)=\hbar \omega_{z} \kappa^{2} \epsilon^{2}\left[S_{n n}^{(-)}\left(\omega_{z}\right)\right] .
$$

Here we have introduced the spectral density of photon number fluctuations $S_{n n}^{( \pm)}(\omega)=$ $2 \bar{n} \kappa\left(\kappa^{2}+(\Delta \pm \omega)^{2}\right)^{-1}[16]$, with $\Delta=\omega_{p}-\omega_{c}^{\prime}$ begin the probe detuning from the atoms shifted cavity resonance.

The second term in Eq. 25] accounts for the effect of transient atomic motion on the cavity field. To evaluate this term we take the time average over an atomic oscillation period.

$$
\begin{aligned}
& i \kappa \epsilon\left\langle a^{\dagger}(0)(n(t)-\bar{n}) e^{i \omega_{z} t}-(n(t)-\bar{n}) a(0) e^{-i \omega_{z} t}\right\rangle \\
& =\bar{n} \epsilon^{2} \kappa\left(L\left(\omega_{p}+\omega_{z}\right)-L^{*}\left(\omega_{p}-\omega_{z}\right)+L\left(\omega_{p}-\omega_{z}\right)-L^{*}\left(\omega_{p}+\omega_{z}\right)\right)\left\langle a^{\dagger}(0) a(0)\right\rangle \\
& =\kappa^{2} \epsilon^{2}\left[S_{n n}^{(-)}\left(\omega_{z}\right)-S_{n n}^{(+)}\left(\omega_{z}\right)\right]\left\langle a^{\dagger} a\right\rangle .
\end{aligned}
$$


These terms represent cavity cooling/anti-cooling. In total, the change in energy is,

$$
\frac{d}{d t} E=\hbar \omega_{z} \kappa^{2} \epsilon^{2}\left[S_{n n}^{(-)}\left(\omega_{z}\right)+\left(S_{n n}^{(-)}\left(\omega_{z}\right)-S_{n n}^{(+)}\left(\omega_{z}\right)\right)\left\langle a^{\dagger} a\right\rangle\right] .
$$

\section{Measuring backaction heating by the evaporative loss of trapped atoms}

The accuracy of our measurement depends on assumptions made in interpreting the observed transmission lineshapes, several of which we verified experimentally. For example, we examined the dynamics of evaporative cooling in the atomic medium. For this, we interrupted the cavity transmission measurement, released the atoms from the intracavity optical trap and imaged them $4 \mathrm{~ms}$ later to measure their temperature. Within our measurement resolution of $0.1 \mu \mathrm{K}$, this temperature remained constant. Thus, our quantification of heating through the rate of atom loss is valid. Furthermore, by extinguishing the cavity probe

light momentarily during cavity probing, and comparing the cavity transmission when the probe was turned off and then turned on again, we determined a timescale of $3 \mathrm{~ms}$ for $N$ to equilibrate by evaporative cooling following an increase of thermal energy of the collective mode. Since this timescale is short compared to the $\simeq 100 \mathrm{~ms}$ span of the resonant transmission signal, we are justified in using simultaneous measurements of $d N / d t$ and $\bar{n}$ to determine the instantaneous heating rate.

To interpret our measurements as relating to the quantum nature of the intracavity field, it was necessary to establish that quantum fluctuations dominate over classical, technical intensity fluctuations which would also lead to heating [38]. For this, we measured the lightinduced heating for varying probe intensities, with $\bar{n}$ at the cavity resonance ranging from $\bar{n}=0.2$ to 20 . Noting that the contribution of quantum fluctuations to the atom heating rate scales as $\bar{n}$ while that of technical fluctuations scales as $\bar{n}^{2}$, we find that technical fluctuations account for less than $10 \%$ of the atom heating rate at the light level used for Figs. 2 and 3.

\section{Visibility of photon fluctuations outside the cavity}

In this section, we provide support for the well-established[39] but oft-forgotten result that the spectrum of intra-cavity quantum fluctuations of the photon number is not visible in light transmitted through the coherently driven cavity. For a two sided cavity we have 
[37]:

$$
\begin{aligned}
c_{\text {out }}(t)+c_{\text {in }}(t) & =\sqrt{\kappa} b(t), \\
d_{\text {out }}(t)+d_{\text {in }}(t) & =\sqrt{\kappa} b(t), \\
b(\omega) & =\frac{\sqrt{\kappa} c_{i n}(\omega)+\sqrt{\kappa} d_{i n}(\omega) .}{\kappa-i\left(\omega-\omega_{c}^{\prime}\right)} .
\end{aligned}
$$

The operators $c_{\text {out }}, d_{\text {out }}, c_{i n}, d_{\text {in }}$ are photon annihilation operators for the outgoing and ingoing fields on either side of the cavity, and $b$ is again the cavity field annihilation operator. The known commutation relations are,

$$
\left[c_{i n}\left(\omega_{1}\right), c_{i n}^{\dagger}\left(\omega_{2}\right)\right]=\delta\left(\omega_{1}-\omega_{2}\right), \quad\left[c_{i n}\left(t_{1}\right), c_{i n}^{\dagger}\left(t_{2}\right)\right]=\delta\left(t_{1}-t_{2}\right),
$$

and similarly for $d_{i n}$. To examine the spectrum of photon fluctuations inside the cavity we calculate the commutation relation for the cavity field operator:

$$
\begin{aligned}
{\left[b\left(t_{1}\right), b^{\dagger}\left(t_{2}\right)\right] } & =\int \frac{d \omega_{1} d \omega_{2}}{2 \pi} \frac{e^{-i \omega_{1} t_{1}}}{\kappa-i\left(\omega_{1}-\omega_{c}^{\prime}\right)} \frac{e^{i \omega_{2} t_{2}}}{\kappa+i\left(\omega_{2}-\omega_{c}^{\prime}\right)} \times \\
& =\int \frac{d \omega_{1} d \omega_{2}}{2 \pi} \frac{e^{-i \omega_{1} t_{1}}}{\kappa-i\left(\omega_{1}-\omega_{c}^{\prime}\right)} \frac{e^{i \omega_{2} t_{2}}}{\kappa+i\left(\omega_{2}-\omega_{c}^{\prime}\right)} 2 \kappa \delta\left(\omega_{1}-\omega_{2}\right) \\
& =\int \frac{d \omega}{2 \pi} \frac{2 \kappa}{\kappa^{2}+\left(\omega-\omega_{c}^{\prime}\right)^{2}} e^{i \omega\left(t_{2}-t_{1}\right)} \\
& =e^{i \omega_{c}^{\prime}\left(t_{2}-t_{1}\right)-\kappa\left|t_{2}-t_{1}\right|} .
\end{aligned}
$$

From this we obtain the two-time correlation in Eq. 26. Now, for the cavity output, (say, $\left.d_{\text {out }}\right)$,

$$
\begin{aligned}
& {\left[d_{\text {out }}\left(t_{1}\right), d_{\text {out }}^{\dagger}\left(t_{2}\right)\right]=\int \frac{d \omega_{1} d \omega_{2}}{2 \pi} e^{i \omega_{1} t_{1}+i \omega_{2} t_{2}}\left[\frac{\kappa c_{\text {in }}\left(\omega_{1}\right)+\kappa d_{\text {in }}\left(\omega_{1}\right)}{\kappa-i\left(\omega_{1}-\omega_{c}^{\prime}\right)}-d_{\text {in }}\left(\omega_{1}\right),\right.} \\
& \left.\frac{\kappa c_{i n}^{\dagger}\left(\omega_{2}\right)+\kappa d_{i n}^{\dagger}\left(\omega_{2}\right)}{\kappa+i\left(\omega_{2}-\omega_{c}^{\prime}\right)}-d_{i n}^{\dagger}\left(\omega_{2}\right)\right] \\
& =\int \frac{d \omega_{1} d \omega_{2}}{2 \pi} e^{i \omega_{1} t_{1}+i \omega_{2} t_{2}}\left(\frac{2 \kappa^{2} \delta\left(\omega_{1}-\omega_{2}\right)}{\left(\kappa-i\left(\omega_{1}-\omega_{c}^{\prime}\right)\right)\left(\kappa+i\left(\omega_{2}-\omega_{c}^{\prime}\right)\right)}-\right. \\
& \left.\frac{\kappa \delta\left(\omega_{1}-\omega_{2}\right)}{\kappa-i\left(\omega_{1}-\omega_{c}^{\prime}\right)}-\frac{\kappa \delta\left(\omega_{1}-\omega_{2}\right)}{\kappa+i\left(\omega_{2}-\omega_{c}^{\prime}\right)}\right) \\
& =\int \frac{d \omega}{2 \pi} e^{i \omega\left(t_{2}-t_{1}\right)}=\delta\left(t_{1}-t_{2}\right) .
\end{aligned}
$$


The commutation relations for fields outside the cavity are the same as for light entering the cavity, and do not carry any evidence of the photon number dynamics (Eq. 39) inside the cavity.

[1] K. Schwab and M. Roukes, Physics Today July, 36 (2005).

[2] V. Braginskii and F. Y. Khalili, Quantum Measurement (Cambridge University Press, Cambridge, 1995).

[3] S. Gupta et al., Phys. Rev. Lett. 99, 213601 (2007).

[4] Y. Colombe et al., Nature 450, 272 (2007).

[5] F. Brennecke et al., Nature 450, 268 (2007).

[6] D. Kleckner and D. Bouwmeester, Nature 444, 75 (2006).

[7] M. Poggio et al., Phys. Rev. Lett. 99, 017201 (2007).

[8] A. Naik et al., Nature 443, 193 (2006).

[9] S. Gigan et al., Nature 444, 67 (2006).

[10] O. Arcizet et al., Nature 444, 71 (2006).

[11] J. D. Thompson et al., preprint, arXiv: p. 07071724 (2007).

[12] A. Schliesser et al., Phys. Rev. Lett. 97, 243905 (2006).

[13] X. M. H. Huang et al., Nature 421, 496 (2003).

[14] P. Horak et al., Phys. Rev. Lett. 79, 4974 (1997).

[15] V. Vuletić and S. Chu, Phys. Rev. Lett. 84, 3787 (2000).

[16] F. Marquardt et al., Phys. Rev. Lett. 99, 093902 (2007).

[17] I. Wilson-Rae et al., Phys. Rev. Lett. 99, 093901 (2007).

[18] D. Vitali et al., J Opt Soc Am B 20, 1054 (2003).

[19] I. Bouchoule et al., Phys. Rev. A 59, R8 (1999).

[20] D. Harber et al., Phys. Rev. A 72, 033610 (2005).

[21] U. Mohideen and A. Roy, Phys. Rev. Lett. 81, 4549 (1998).

[22] H. Mabuchi et al., Optics Letters 21, 1393 (1996).

[23] C. Hood et al., Science 287, 1447 (2000).

[24] A. Dorsel et al., Phys. Rev. Lett. 51, 1550 (1983).

[25] C. Caves, Phys. Rev. D 23, 1693 (1981). 
[26] J. Dalibard and C. Cohen-Tannoudji, J. Opt. Sci. Am. B 2, 1707 (1985).

[27] J. P. Gordon and A. Ashkin, Phys. Rev. A 21, 1606 (1980).

[28] P. Maunz et al., Phys. Rev. Lett. 94, 033002 (2005).

[29] P. Münstermann et al., Phys. Rev. Lett. 82, 3791 (1999).

[30] R. Loudon and P. Knight, J. Mod. Opt. 34, 709 (1987).

[31] A. Kuzmich, N. P. Bigelow, and L. Mandel, Europhysics Letters 42, 481 (1998).

[32] Y. Takahashi et al., Phys. Rev. A 60, 4974 (1999).

[33] I. Bouchoule and K. Mølmer, Phys. Rev. A 66, 043811 (2002).

[34] M. Auzinsh et al., Phys. Rev. Lett. 93, 173002 (2004).

[35] J. Hald et al., Phys. Rev. Lett. 83, 1319 (2000).

[36] J.M. Geremia, J. K. Stockton and H. Mabuchi, Science 304, 270 (2004).

[37] D. Walls and G. Milburn, Quantum optics (Springer, New York, 1994).

[38] M. Gehm et al., Phys. Rev. A 58, 3914 (1998).

[39] H. Carmichael, An Open Systems Approach to Quantum Optics (Springer-Verlag, New York, 1991). 